\title{
Growth of Pseudomorphic GeSn at Low Pressure with Sn Composition of $16.7 \%$
}

\author{
Joshua Grant ${ }^{1}$, Grey Abernathy ${ }^{1,2}$, Oluwatobi Olorunsola 1,2, Solomon Ojo 1,2 ${ }^{\mathbb{D}}$, Sylvester Amoah ${ }^{1}$, \\ Emmanuel Wanglia ${ }^{1,2}$, Samir K. Saha ${ }^{3}$, Abbas Sabbar ${ }^{1} \mathbb{D}$, Wei Du ${ }^{4}$, Murtadha Alher ${ }^{1,5}$, Bao-Hua Li $^{6}$ and \\ Shui-Qing Yu 1,7,*
}

1 Department of Electrical Engineering, University of Arkansas, Fayetteville, AR 72701, USA; jmg029@uark.edu (J.G.); gabernat@uark.edu (G.A.); ogolorun@uark.edu (O.O.); soojo@uark.edu (S.O.); samoah@email.uark.edu (S.A.); eswangil@uark.edu (E.W.); afsabbar@uark.edu (A.S.); maalher@uark.edu (M.A.)

2 Microelectronics-Photonics Program, University of Arkansas, Fayetteville, AR 72701, USA

3 Department of Physics, University of Arkansas, Fayetteville, AR 72701, USA; sksaha@uark.edu

4 Department of Electrical Engineering and Physics, Wilkes University, Wilkes-Barre, PA 18766, USA; wei.du@wilkes.edu

5 Mechanical Engineering Department, University of Kerbala, Kerbala 56001, Iraq

6 Arktonics, LLC, 1339 South Pinnacle Drive, Fayetteville, AR 72701, USA; arktonics@gmail.com

7 Institute for Nanoscience and Engineering, University of Arkansas, Fayetteville, AR 72701, USA

* Correspondence: syu@uark.edu

Citation: Grant, J.; Abernathy, G.; Olorunsola, O.; Ojo, S.; Amoah, S.; Wanglia, E.; Saha, S.K.; Sabbar, A.; Du, W.; Alher, M.; et al. Growth of Pseudomorphic GeSn at Low Pressure with Sn Composition of 16.7\%. Materials 2021, 14, 7637. https://doi.org/10.3390/ma14247637

Academic Editor: Bruce B. Claflin

Received: 30 October 2021

Accepted: 8 December 2021

Published: 11 December 2021

Publisher's Note: MDPI stays neutral with regard to jurisdictional claims in published maps and institutional affiliations.

Copyright: (C) 2021 by the authors. Licensee MDPI, Basel, Switzerland. This article is an open access article distributed under the terms and conditions of the Creative Commons Attribution (CC BY) license (https:// creativecommons.org/licenses/by/ $4.0 /)$.

\begin{abstract}
Group-IV alloy GeSn holds great promise for the high-performance optoelectronic devices that can be monolithically integrated on Si for near- and mid-infrared applications. Growth of GeSn using chemical vapor deposition technique with various $\mathrm{Sn}$ and Ge precursors has been investigated worldwide. To achieve relatively high Sn incorporation, the use of higher pressure and/or higher order Ge hydrides precursors were reported. In this work, we successfully demonstrated the growth of high-quality GeSn with Sn composition of $16.7 \%$ at low pressure of 12 Torr. The alloy was grown using the commercially available $\mathrm{GeH}_{4}$ and $\mathrm{SnCl}_{4}$ precursors via a chemical vapor deposition reactor. Material and optical characterizations were performed to confirm the Sn incorporation and to study the optical properties. The demonstrated growth results reveal a low-pressure growth window to achieve high-quality and high Sn alloys for future device applications.
\end{abstract}

Keywords: group-IV; GeSn; CVD growth; low pressure

\section{Introduction}

GeSn alloys offer promising optical advantages compared to traditional group-IV semiconductor materials such as Si and Ge [1-3]. With sufficiently high Sn composition, the true direct bandgap GeSn has led to the successful demonstration of optically pumped and electrically injected GeSn lasers [4-8]. The tunable bandgap covering broad nearand mid-infrared wavelength enables the development of light emitter and detectors towards Si-based longwave integrated optoelectronics (LIO) applications [9-12]. Moreover, the complementary metal-oxide-semiconductor (CMOS) process compatibility allows GeSn-based devices to be monolithically integrated on Si substrates [13-15].

Growth of GeSn alloys on Si substrate is difficult because of the low solubility ( $<1 \%)$ of $\mathrm{Sn}$ in $\mathrm{Ge}$, the instability of $\alpha$-Sn above $13^{\circ} \mathrm{C}$, and the large lattice mismatch between $\mathrm{GeSn}$ and $\mathrm{Si}(>4.2 \%)$. To address these challenges, non-equilibrium condition growth techniques were developed. Low temperature growth using molecular-beam epitaxy (MBE) [16-18] and chemical vapor deposition (CVD) [19-21] have been reported. For the past decade, the CVD growth technique has been increasingly investigated worldwide. The early growth utilized deuterium-stabilized stannane $\left(\mathrm{SnD}_{4}\right)$ as the Sn precursor $[22,23]$, which suffered from high-cost and instability, and motivated the use of low cost, stable, and 
commercially available tin-tetrachloride $\left(\mathrm{SnCl}_{4}\right)$. It has been reported that the $\mathrm{SnCl}_{4}$-based growth achieves device-level material quality $[20,24]$. Additionally, using low cost and commercially available germane $\left(\mathrm{GeH}_{4}\right)$ as Ge precursor was widely reported [25-28].

From the device application perspective, higher $\mathrm{Sn}$ incorporation is desired due to: (i) more bandgap directness enhances the efficiency of emitter; and (ii) an extended spectral response cutoff is in favor of long wavelength detection. To achieve higher Sn composition, higher order Ge hydrides, such as $\mathrm{Ge}_{2} \mathrm{H}_{6}$ and $\mathrm{Ge}_{3} \mathrm{H}_{8}$, were adopted due to their favorable decomposition at low temperature [29-33]. Alternatively, plasma-enhanced CVD growth was successfully demonstrated in an ultra-high vacuum (UHV) chamber [34]. Currently, the $\mathrm{Sn}$ incorporation is a figure of merit from the material growth perspective. The summary of GeSn CVD growth results using $\mathrm{SnCl}_{4}$ as $\mathrm{Sn}$ precursor is shown in Table 1.

Table 1. Summary of GeSn growth using $\mathrm{SnCl}_{4}$ via CVD reactor.

\begin{tabular}{|c|c|c|c|c|c|}
\hline $\begin{array}{c}\mathrm{Ge} \\
\text { Precursor }\end{array}$ & $\begin{array}{l}\text { Pressure } \\
\text { (Torr) }\end{array}$ & $\begin{array}{c}\mathrm{SnCl}_{4} \text { Flow } \\
\text { Ratio }\end{array}$ & $\begin{array}{c}\text { Temperature } \\
\left({ }^{\circ} \mathrm{C}\right)^{1}\end{array}$ & $\begin{array}{c}\mathrm{Sn} \\
\text { Incorporation }\end{array}$ & Ref. \\
\hline \multirow{8}{*}{$\mathrm{GeH}_{4}$} & \multirow{3}{*}{2} & 0.0025 & $250-270$ & $2.1-8.7 \%$ & [25] \\
\hline & & 0.005 & 270 & $6.0 \%$ & [25] \\
\hline & & 0.01 & $240-350$ & $1.5-7.9 \%$ & [25] \\
\hline & \multirow{2}{*}{40} & 0.0085 & $290-350$ & $2.5-9.1 \%$ & [28] \\
\hline & & 0.012 & 290-350 & $3.0-10.0 \%$ & [28] \\
\hline & \multirow{2}{*}{50} & 0.0006 & $325-335$ & $6.0-7.0 \%$ & [27] \\
\hline & & 0.0008 & 335 & $5.0 \%$ & [27] \\
\hline & 120 & 0.0007 & $280-320$ & $8.0-18.0 \%$ & [26] \\
\hline \multirow{6}{*}{$\mathrm{Ge}_{2} \mathrm{H}_{6}$} & N.A. & 0.0045 & $340-400$ & $5.0-14.0 \%$ & [30] \\
\hline & 45 & 0.01 & $375-475$ & $3.5-10.0 \%$ & [29] \\
\hline & \multirow{3}{*}{100} & $0.02-0.04$ & 300 & $9.9-10.6 \%$ & [31] \\
\hline & & $0.027-0.05$ & 320 & $5.8-7.8 \%$ & [31] \\
\hline & & 0.0532 & $301-349$ & $6.0-15.0 \%$ & {$[32,33]$} \\
\hline & 760 & 0.04 & 320 & $6.6 \%$ & [31] \\
\hline $\begin{array}{c}\text { This work } \\
\mathrm{GeH}_{4}\end{array}$ & 12 & 0.0025 & 260 & $16.7 \%$ & \\
\hline
\end{tabular}

${ }^{1}$ Lower temperature has the higher Sn incorporation.

It is generally acknowledged that using higher order Ge hydrides precursors would facilitate $\mathrm{Sn}$ incorporation. From Table 1, it can be seen that most higher Sn growth work $\left(>10.0 \%\right.$ ) were accomplished by using $\mathrm{Ge}_{2} \mathrm{H}_{6}$. Moreover, the chamber pressure plays an important role for $\mathrm{Sn}$ incorporation, as generally the high $\mathrm{Sn}$ materials are grown under higher pressure. The maximum Sn composition of $18.0 \%$ was achieved under relatively high pressure of 120 Torr [26]. Despite employing $\mathrm{Ge}_{2} \mathrm{H}_{6}$ or $\mathrm{Ge}_{3} \mathrm{H}_{8}$ offers a viable solution for high $\mathrm{Sn}$ material growth, using $\mathrm{GeH}_{4}$ is preferred in manufacturing due to its much lower cost; it is worth noting that higher Sn alloys may suffer from deteriorated material quality, limiting the device performance using such material. Therefore, a growth method that meets utilizing industry-preferred precursors, higher Sn incorporation, and devicelevel material quality is highly desired, which has yet been fully explored.

In this work, we demonstrate the growth of a GeSn alloy with Sn composition of $16.7 \%$. The material was grown using commercially available $\mathrm{GeH}_{4}$ and $\mathrm{SnCl}_{4}$ precursors via a home-built UHV-CVD reactor. The pressure was kept as low as 12 Torr. Material and optical characterizations were conducted to confirm the Sn incorporation as well as to show optical properties. In addition, the surface defects were analyzed to speculate the growth dynamic. While the in-depth understanding of low-pressure growth is still undergoing, the grown GeSn alloy presented in this work indicates a new growth window towards high Sn and high material quality for future device applications. 


\section{Experimental Methods}

The GeSn sample was grown on a 100-mm p-type silicon (100) substrate with the resistivity of $10-20 \Omega \cdot \mathrm{cm}$ via a custom built cold walled UHV-CVD reactor. The base pressure of the reactor is $10^{-10}$ Torr. The silicon substrate was cleaned using a piranha etch solution $\left(\mathrm{H}_{2} \mathrm{SO}_{4}: \mathrm{H}_{2} \mathrm{O}_{2}=1: 1\right)$, followed by oxide removal and hydrogen passivation solution using the diluted HF. Prior to GeSn film growth, a Ge buffer layer was grown at 1 Torr using a two-step process: the first step growth was performed at $375^{\circ} \mathrm{C}$ while the second step was at $600{ }^{\circ} \mathrm{C}$. The flow rate of Ge precursor $\left(\mathrm{GeH}_{4}\right)$ during the second step of the buffer growth was reduced by half of that in the first step. The growth rate is $0.2 \mu \mathrm{m} / \mathrm{min}$ and the overall thickness is $6.6 \mu \mathrm{m}$. The GeSn layer growth was performed at $260{ }^{\circ} \mathrm{C}$ using the $\mathrm{GeH}_{4}$ and $\mathrm{SnCl}_{4}$ as $\mathrm{Ge}$ and $\mathrm{Sn}$ precursors, respectively, with the argon (Ar) acted as the carrier gas. The growth rate was estimated as $\sim 1.33 \mathrm{~nm} / \mathrm{min}$.

Material characterizations were performed following the growth. Atomic force microscopy (AFM) was used to investigate the surface morphology. The high-resolution X-ray diffraction (XRD) 20- $\omega$ scan along the $\mathrm{Si}$ (004) plane and the reciprocal space map (RSM) along $\overline{22} 4$ were employed to identify the Sn composition, the degree of strain, and the layer thickness. The transmission electron microscopy (TEM) image was obtained to cross-check the layer thickness, as well as to check the material quality.

Optical characterizations were performed to further study the GeSn thin film properties. A Raman spectroscopy setup including a $632 \mathrm{~nm}$ HeNe laser pump source by Thor Labs (Newton, NJ, USA) at $5 \mathrm{~mW}$ power and a Horiba iHR 550 (Kisshoin, Minami-ku, Kyoto, Japan) grating-based spectrometer with a charge coupled device (CCD) detector was employed to quantify the shift of longitudinal optical (LO) phonon peak. A Ge bulk sample was used as reference. The absorption coefficient spectrum was measured via a J. A. Woollam V-Vase ellipsometer (Lincoln, NE, USA) in the range of 0.496 to $4.768 \mathrm{eV}$ ( 260 to $2500 \mathrm{~nm}$ ) with a resolution of $10 \mathrm{~nm}$ at incidence angle of $70^{\circ}$. The absorption coefficient was obtained by using the Johs-Herzinger model, and then was fitted by applied physical model [35]. The photoluminescence (PL) study was conducted using the standard off-axis setup configuration and lock-in techniques. A $1064 \mathrm{~nm}$ pulsed laser was used as the pumping source. The emissions were collected using a Horiba iHR 320 (Kisshoin, Minami-ku, Kyoto, Japan) grating based spectrometer equipped with a PbS detector with a spectral cutoff at $3.0 \mu \mathrm{m}$.

\section{Results and Discussion}

Figure 1a shows the optical image of the entire wafer that was taken immediately after the growth was completed. Two distinct regions were observed: a shiny region with some island-like areas at the center (region I), surrounded by a cloudy region (outer ring, region II). The clearly-resolved regions suggest different material quality. Generally speaking, the shiny region indicates a higher quality which will be confirmed via the following material characterizations. Note that there is a "transition" area between the regions I and II, showing a bit different color. As this area features similar surface roughness and morphology after characterization, it was included in region II.

AFM images of $0.5 \mu \mathrm{m} \times 0.5 \mu \mathrm{m}$ area are shown in Figure $1 \mathrm{~b}, \mathrm{c}$ for regions I and II, respectively. Region I features surface roughness of $\sim 3 \mathrm{~nm}(-1.5$ to $1.4 \mathrm{~nm})$ compared to that of region II of $\sim 30 \mathrm{~nm}(-15.8$ to $13.3 \mathrm{~nm})$. The smaller surface roughness indicates a better material quality in region I. The rough surface in region II may be due to the $\mathrm{Sn}$ segregation, which was observed in previous study [36].

High-resolution XRD was used to examine crystalline characteristics of the sample. Figure 2a shows the $2 \theta-\omega$ scan of Region I. The well-resolved peaks at $69.1^{\circ}$ and $66.1^{\circ}$ correspond to Si substrate and Ge buffer, respectively. Based on previous study, incorporation of Sn would shift the peak towards lower angle. Therefore, the peak at $63.1^{\circ}$ is assigned to GeSn thin film. The multiple oscillations between $63^{\circ}$ and $65^{\circ}$ are Pendellösung fringes, which indicates uniformed Sn incorporation and a smooth interface between GeSn and Ge 
buffer. Moreover, via XRD simulation, the Sn composition and film thickness were fitted as $16.7 \%$ and $40 \mathrm{~nm}$, respectively.
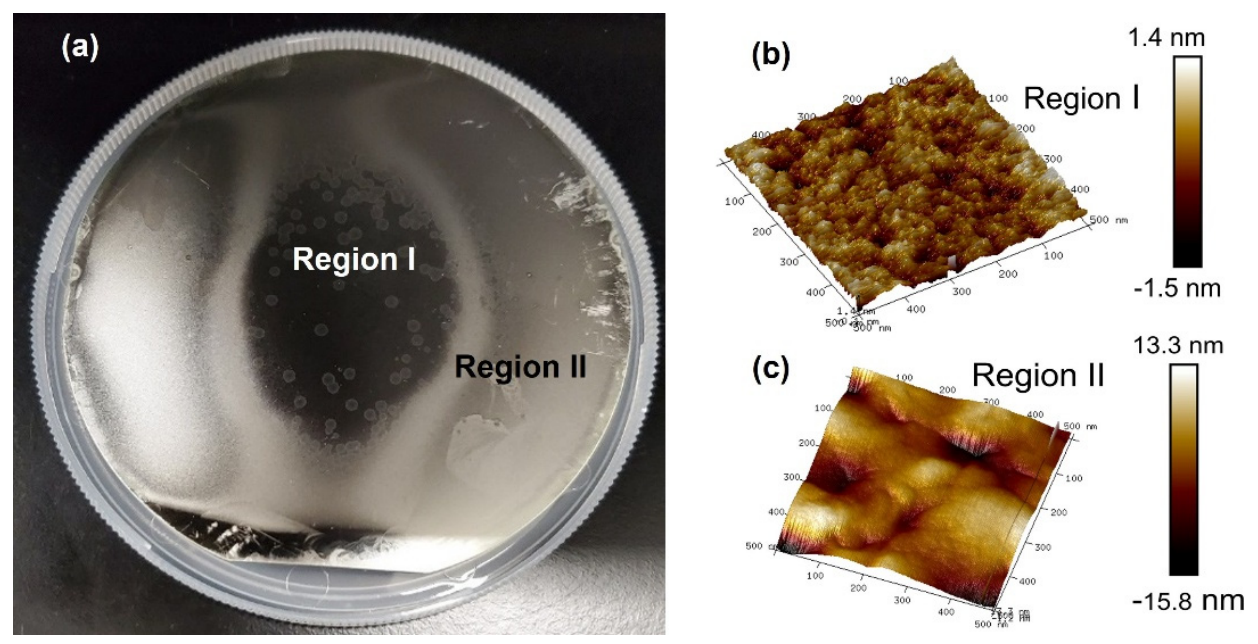

Figure 1. (a) Optical image of as grown sample with two distinct regions (Region I: shiny center, and region II: cloudy outer ring). AFM images of (b) region I and (c) region II showing the surface roughness of $\sim 3 \mathrm{~nm}$ and $\sim 30 \mathrm{~nm}$, respectively, indicating a better quality of region $\mathrm{I}$.
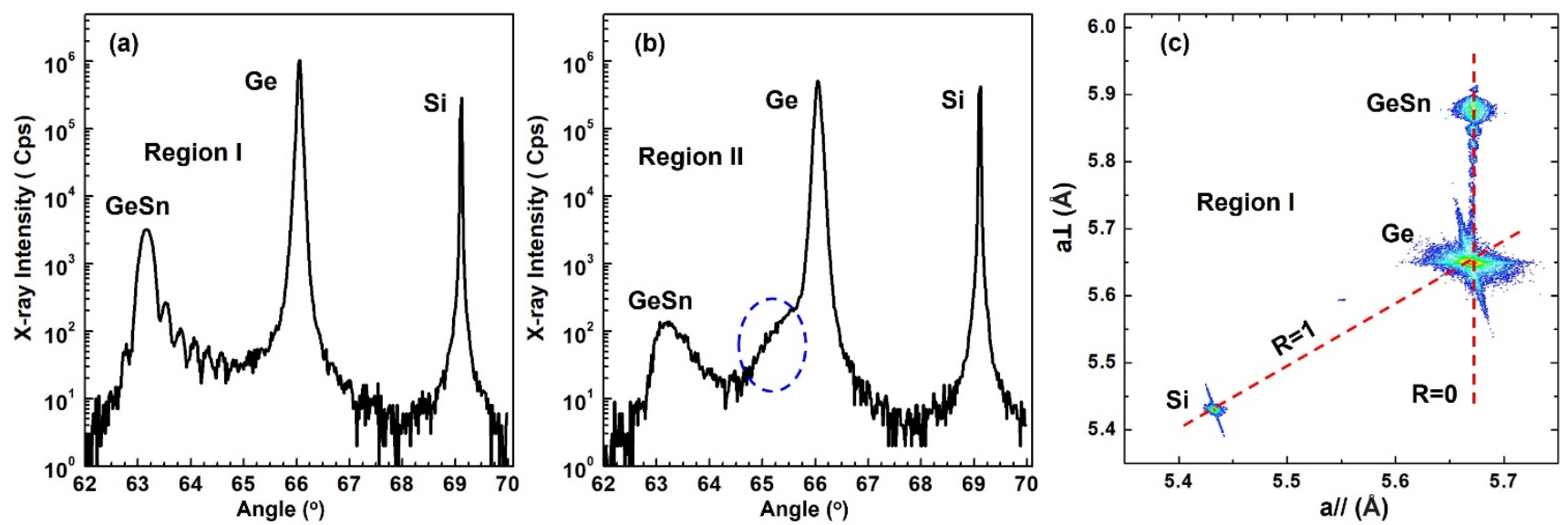

Figure 2. (a,b) XRD 20-w scans of GeSn film on Ge-buffered Si (100) of region I and II. (c) RSM contour plot of region I showing the pseudomorphic growth.

The $2 \theta-\omega$ scan of Region II is shown in Figure $2 b$. The Si substrate and Ge buffer peaks are the same as in Figure 2a. For the GeSn film, the peak position at $\sim 63.3^{\circ}$ is a slightly higher than that in (a). However, the peak linewidth is much broader, indicating the lower material quality of region II compared to region I. The dramatically reduced signal intensity (1.5 order of magnitudes) and the disappearance of Pendellösung fringes also suggest the lower material quality. In addition, two distinct features can be observed in Figure 2b: (i) asymmetric GeSn peak with smaller slope at higher angle side; and (ii) A clear shoulder at $\sim 65^{\circ}$ implying the possible existence of a GeSn peak close to Ge reference peak. This can be interpreted as the Sn segregation near the surface, which decreases the Sn incorporation down to less than 3\% locally, resulting in an additional GeSn layer with lower $\mathrm{Sn}$ composition. At current stage, it is very difficult to accurately position this GeSn layer. Due to the Sn segregation, the Sn composition in region II is a little lower than that in region $\mathrm{I}$.

Figure $2 \mathrm{c}$ shows the RSM contour plot of region I. The in-plane $(a / /)$ and out-of-plane $(a \perp)$ lattice constants were extracted from XRD simulation. " $\mathrm{R}=1$ " represents relaxation line and " $\mathrm{R}=0$ " indicates the pseudomorphic growth line. It is clear that the Ge buffer is 
almost strain relaxed while the GeSn thin film is lattice-matched to Ge buffer. By using the Vegard's law: $a_{G e_{1-x} S n_{x}}=(1-x) a_{G e}+x a_{S n}$, where lattice constants for Ge and Sn are 5.646 and $6.489 \AA$, respectively, the degree of strain can be calculated. The Ge buffer is under a slight tensile strain of $0.17 \%$, and the GeSn film experiences compressive strain of $2.03 \%$.

The sample was further characterized using TEM technique. The cross-sectional image of region I is shown in Figure 3. The interface between GeSn layer and Ge buffer can be clearly resolved (dashed line), where the relatively low density of threading dislocations was observed. Most threading dislocations were localized at GeSn/Ge interface and did not propagate to GeSn layer, resulting in high material quality. The thickness of GeSn was measured as $42 \mathrm{~nm}$, matching with the result obtained from XRD.

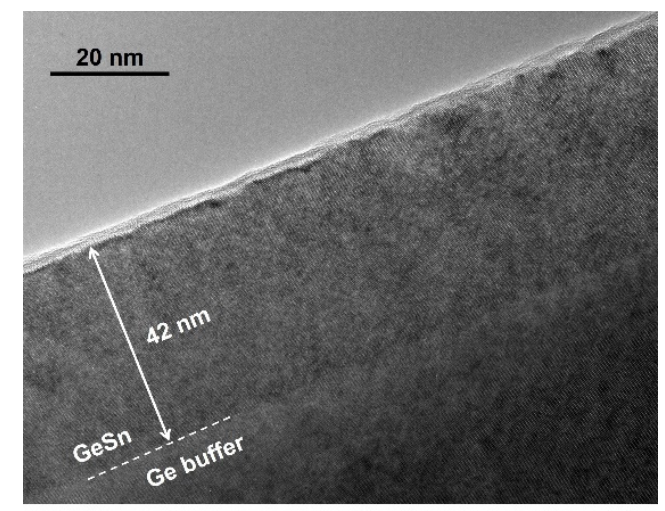

Figure 3. Cross-sectional TEM image showing the GeSn layer thickness of $42 \mathrm{~nm}$.

The optical properties of GeSn thin film were further studied. Figure 4a shows the Raman spectroscopy of the two regions. A Ge bulk sample was used to calibrate the measurement setup, whose Ge-Ge LO phonon peak locates at $300 \mathrm{~cm}^{-1}$. For GeSn thin film, the Ge-Ge LO peaks were obtained at 296.4 and $298.2 \mathrm{~cm}^{-1}$ for regions I and II, respectively. The peak shift towards lower wavenumber is due to the induced strain by incorporation of Sn atoms into Ge lattice. The more Sn incorporated; the more peak shift can be observed. Therefore, region I features a slightly higher Sn incorporation than region II, which matches the XRD 20- $\omega$ scan measurement results shown in Figure 2.
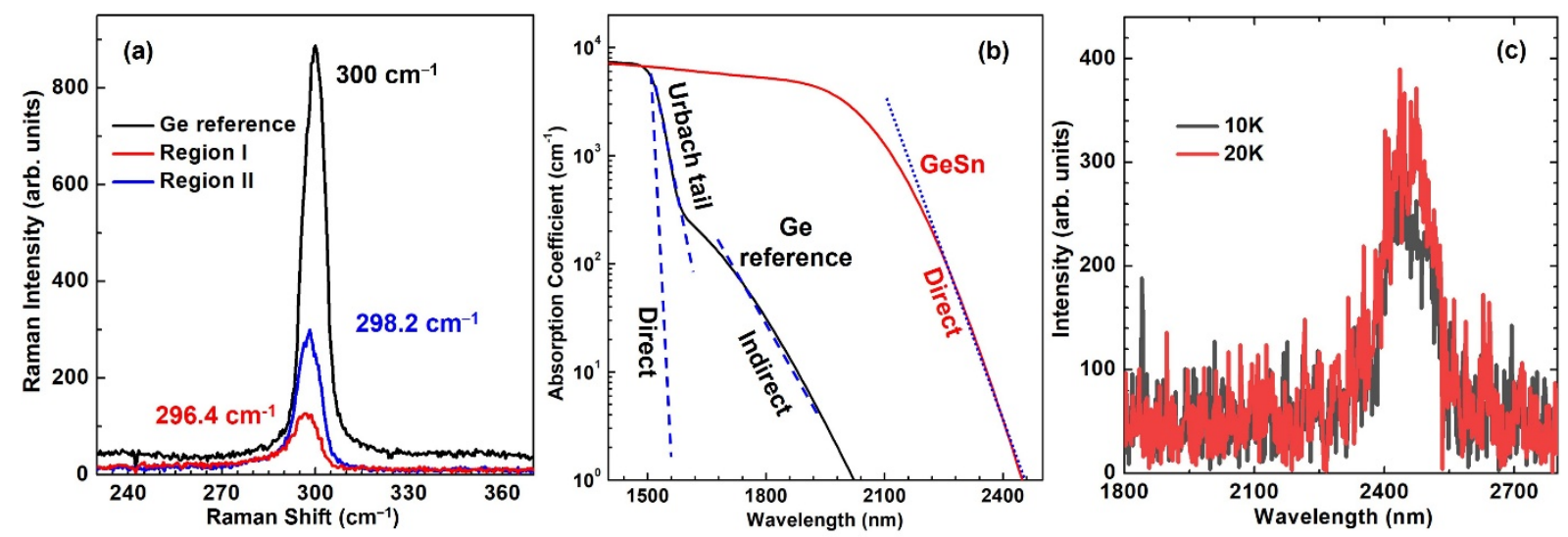

Figure 4. (a) Raman spectroscopy showing the shift of Ge-Ge LO phonon peak. (b) Spectral absorption coefficient measured using ellipsometry. (c) PL spectra at 10 and $20 \mathrm{~K}$.

The spectral absorption coefficient of region I was measured using ellipsometry spectroscopy, as shown in Figure 4b. The absorption curve of Ge was also plotted as a reference. Compared to Ge, the measured absorption curve shows significant red-shift, indicating 
the origin of absorption is from GeSn layer. For Ge, since the energy separation between $\Gamma$ and $L$ valleys is $\sim 140 \mathrm{meV}$, the absorption associated with direct bandgap, Urbach tail and indirect bandgap can be identified and fitted using the method described by Tran, et al. [35], as shown as dashed lines in Figure 4b. For GeSn, it has been reported that the pseudomorphic GeSn on Ge may have a direct bandgap at Sn composition of $\sim 17 \%$. Therefore, the $\Gamma$ and $L$ valley separation in this $16.7 \%$ Sn sample may be only a few meV. As a result, the indirect absorption is considerably weak and cannot be resolved. The direct bandgap absorption was fitted with the wavelength cut-off at $\sim 2460 \mathrm{~nm}$ (dotted line). Note that the indirect bandgap energy extracted from fitting is not accurate due to the weak indirect absorption. The ellipsometry measurement in this work focuses on the estimation of direct band edge.

Figure $4 \mathrm{c}$ shows the PL spectra of region I at 10 and $20 \mathrm{~K}$. The PL peak is observed at $\sim 2450 \mathrm{~nm}$, corresponding to the bandgap energy of $0.506 \mathrm{eV}$. The measured PL peak is in good agreement with ellipsometry measurement shown in Figure $4 \mathrm{~b}$. Note that according to theoretical calculation, the bandgap energy of relaxed $16.7 \%$ Sn alloy in $\Gamma$ valley is between 0.32 and $0.42 \mathrm{eV}$ at room temperature, depending on the selected bowing parameter. Moreover, the compressive strain will shift conduction band edge upwards while valence band downwards, and consequently will increase the bandgap energy for a few tens of meV [37]. Therefore, the measured PL peak energy of $0.506 \mathrm{eV}$ at $10 \mathrm{~K}$ is consistent with the bandgap calculation using abovementioned method. On the other hand, the relative low intensity of PL peak at low temperature suggests that the material quality can be improved by optimizing the growth recipe in low pressure growth window. Moreover, the low peak intensity is also attributed to weak absorption with thin active GeSn layer $(40 \mathrm{~nm})$.

Optical image in Figure 5a shows a few pyramidal defects in region I. To further understand the origin of the defects, the scanning electron microscope (SEM) image was taken using a FEI Nova Nanolab 200 (Hillsboro, OR, USA) to probe the details. These defects are originated from Ge buffer layer as islands during the material growth [38]. The initial islands are formed as microscale pyramidal defects, which then propagate to GeSn layer surface due to that the growth rate of Ge buffer not being low enough $(0.2 \mu \mathrm{m} / \mathrm{min})$ to suppress the formation of islands, resulting in 3-dimentional growth dominated by Volmer-Weber growth mechanism instead of desired Stranski-Krastanov growth mechanism [39]. The formation of pyramidal defects is believed to be caused by contamination from hydrogen clusters at the Si substrate/Ge buffer interface. These clusters hinder the mobility of Ge adatoms along the sample surface and create favorable nucleation sites in Ge layer. Similar defects were observed and reported elsewhere [31]. Since these defects would act as non-radiative recombination centers and consequently would degrade the device performance, the growth recipe needs to be optimized to reduce the defect density.

(a)

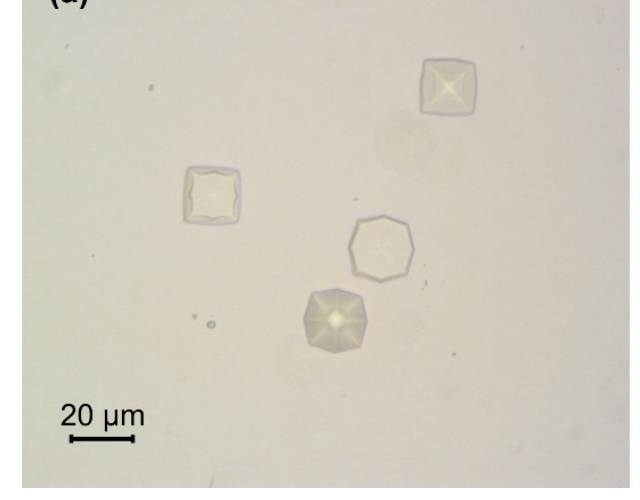

Figure 5. (a) Optical image of defects in region I. (b) SEM image indicating pyramidal island defects in region $\mathrm{I}$.

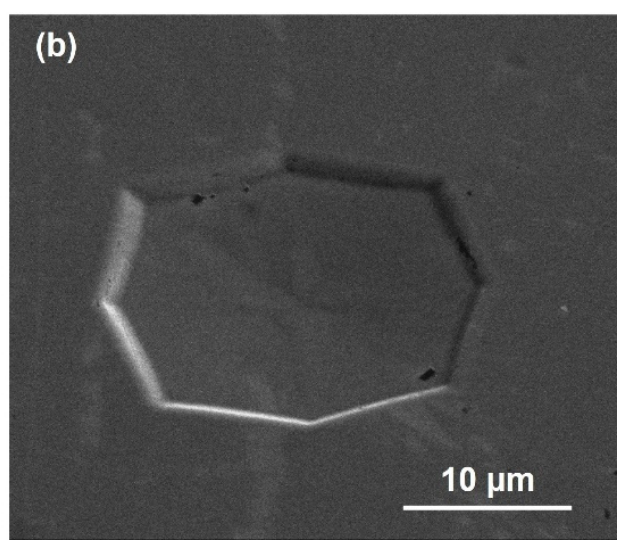


It is worth noting that during the GeSn layer growth, the breakdown of precursors begins at the edge of wafer and continues towards center. At growth temperature of $260{ }^{\circ} \mathrm{C}$, the $\mathrm{SnCl}_{4}$ decomposes completely while the $\mathrm{GeH}_{4}$ does not fully decompose, resulting in the excess of $\mathrm{Sn}$ adatoms over Ge at the edge of wafer. As the precursor gases continue to contact wafer surface the ratio of Sn over Ge is reduced and the formation of Sn agglomerations is suppressed, which improves the material quality near the center of wafer. This leads to two distinct regions shown in Figure 1a, with the higher quality region at the center.

The GeSn growth pressure of 12 Torr was selected in the following way: (i) The breakdown rate of $\mathrm{Ge}$ precursor $\mathrm{GeH}_{4}$ increases as the pressure increases. To obtain the High Sn incorporation without Sn agglomeration at the surface due to Sn overabundance, the pressure of greater than 10 Torr is preferred; (ii) The $\mathrm{Sn}$ precursor $\mathrm{SnCl}_{4}$ used in our UHV-CVD system relies on the vapor pressure of the liquid in the bubbler, which limits the maximum growth pressure to be less than 20 Torr; and (iii) Based on the previous studies [34,36], reducing the $\mathrm{SnCl}_{4}$ partial pressure would facilitate the $\mathrm{Sn}$ incorporation, and therefore the $\mathrm{Ar}$ carrier gas was also used to control the $\mathrm{SnCl}_{4}$ partial pressure. The growth recipe including pressure, temperature, Ge/Sn ratio, etc. can be further optimized to improve the material quality as well as to enhance the $\mathrm{Sn}$ incorporation.

\section{Conclusions}

In this work, a GeSn alloy was grown using commercially available $\mathrm{GeH}_{4}$ and $\mathrm{SnCl}_{4}$ precursors via a home-built UHV-CVD reactor at low pressure of 12 Torr. The Sn composition of $16.7 \%$ was achieved as identified by XRD measurement. The optical characterizations including Raman spectroscopy, ellipsometry spectroscopy, and PL showed the shift of Ge-Ge LO phonon peak, spectral cut-off at $\sim 2460 \mathrm{~nm}$, and emission peak at $\sim 2450 \mathrm{~nm}$ at $20 \mathrm{~K}$, confirming the successfully grown GeSn alloy. Moreover, the analysis of pyramidal defects indicated the Volmer-Weber growth mechanism in Ge buffer. The growth results reported in this work indicate a new low-pressure GeSn growth window.

Author Contributions: Conceptualization, J.G. and S.-Q.Y.; methodology, J.G., G.A. and A.S.; software, J.G.; validation, J.G., G.A., O.O. and S.-Q.Y.; formal analysis, J.G., M.A.; investigation, J.G., G.A., O.O., S.O., S.A., E.W. and S.K.S.; resources, J.G., G.A., O.O., S.O. and E.W.; data curation, J.G., G.A., O.O., S.O., S.A. and E.W.; writing-original draft preparation, J.G., M.A., B.L. and W.D.; writing-review and editing, J.G., W.D., M.A., G.A., O.O., S.O., S.A., E.W., S.K.S., A.S., B.L. and S.-Q.Y.; visualization, J.G. and W.D.; supervision, S.-Q.Y.; project administration, S.-Q.Y.; funding acquisition, S.-Q.Y. All authors have read and agreed to the published version of the manuscript.

Funding: This research was funded by United States Air Force Office of Scientific Research (AFOSR), grant number FA9550-19-1-0341.

Institutional Review Board Statement: Not applicable.

Informed Consent Statement: Not applicable.

Data Availability Statement: Data sharing is not applicable to this article.

Acknowledgments: Wei Du appreciates support from Provost's Research and Scholarship Fund at Wilkes University. The authors would like to acknowledge Mourad Benamara and Andrian Kuchuk from the Institute for Nanoscience and Engineering at the University of Arkansas for their assistance with TEM imaging and XRD measurements.

Conflicts of Interest: The authors declare no conflict of interest.

\section{References}

1. Wirths, S.; Buca, D.; Mantl, S. Si-Ge-Sn alloys: From growth to applications. Prog. Cryst. Growth Charact. Mater. 2016, 62, 1-39. [CrossRef]

2. Kouvetakis, J.; Chizmeshya, A.V.G. New classes of Si-based photonic materials and device architectures via designer molecular routes. J. Mater. Chem. 2007, 17, 1649. [CrossRef]

3. Soref, R. Mid-infrared photonics in silicon and germanium. Nat. Photonics 2010, 4, 495-497. [CrossRef] 
4. Ghetmiri, S.A.; Du, W.; Margetis, J.; Mosleh, A.; Cousar, L.; Conley, B.R.; Domulevicz, L.; Nazzal, A.; Sun, G.; Soref, R.; et al. Direct-bandgap GeSn grown on Silicon with $2230 \mathrm{~nm}$ photoluminescence. Appl. Phys. Lett. 2014, 105, 151109. [CrossRef]

5. Wirths, S.; Geiger, R.; von den Driesch, N.; Mussler, G.; Stoica, T.; Mantl, S.; Ikonic, Z.; Luysberg, M.; Chiussi, S.; Hartmann, J.M.; et al. Lasing in direct-bandgap GeSn alloy grown on Si. Nat. Photon. 2015, 9, 88-92. [CrossRef]

6. Margetis, J.; Al-Kabi, S.; Du, W.; Dou, W.; Zhou, Y.; Pham, T.; Grant, P.; Ghetmiri, S.; Mosleh, A.; Li, B.; et al. Si-based GeSn lasers with wavelength coverage of 2-3 $\mathrm{m}$ and operating temperatures up to $180 \mathrm{~K}$. ACS Photonics 2017, 5, 827-833. [CrossRef]

7. Stange, D.; von den Driesch, N.; Zabel, T.; Armand-Pilon, F.; Rainko, D.; Marzban, B.; Zaumseil, P.; Hartmann, J.M.; Ikonic, Z.; Capellini, G.; et al. GeSn/SiGeSn Heterostructure and Multi Quantum Well Lasers. ACS Photonics 2018, 5, 4628-4636. [CrossRef]

8. Zhou, Y.; Miao, Y.; Ojo, S.; Tran, H.; Abernathy, G.; Grant, J.M.; Amoah, S.; Salamo, G.; Du, W.; Liu, J.; et al. Electrically injected GeSn lasers on Si operating up to 100 K. Optica 2020, 7, 924-928. [CrossRef]

9. Chrétien, J.; Pauc, N.; Armand-Pilon, F.; Bertrand, M.; Thai, Q.-M.; Casiez, L.; Bernier, N.; Dansas, H.; Gergaud, P.; Delamadeleine, E.; et al. GeSn Lasers Covering a Wide Wavelength Range Thanks to Uniaxial Tensile Strain. ACS Photonics 2019, 6, 2462-2469. [CrossRef]

10. Zhou, Y.; Dou, W.; Du, W.; Pham, T.; Ghetmiri, S.; Al-Kabi, S.; Mosleh, A.; Alher, M.; Margetis, J.; Tolle, J.; et al. Systematic study of GeSn heterostructure-based light-emitting diodes towards mid-infrared applications. J. Appl. Phys. 2016, 120, 023102. [CrossRef]

11. Tran, H.; Pham, T.; Margetis, J.; Zhou, Y.; Dou, W.; Grant, P.C.; Grant, J.M.; Al-kabi, S.; Du, W.; Sun, G.; et al. Si-based GeSn photodetectors towards mid-infrared imaging applications. ACS Photonics 2019, 6, 2807-2815. [CrossRef]

12. Wang, W.; Dong, Y.; Lee, S.-Y.; Loke, W.-K.; Lei, D.; Yoon, S.-F.; Liang, G.; Gong, X.; Yeo, Y.-C. Floating-base germanium-tin heterojunction phototransistor for high-efficiency photodetection in short-wave infrared range. Opt. Express 2017, $25,18502$. [CrossRef]

13. Soref, R.; Buca, D.; Yu, S.-Q. Group IV Photonics: Driving Integrated Optoelectronics. Opt. Photonics News 2016, $27,32-39$. [CrossRef]

14. Wang, X.; Liu, J. Emerging technologies in Si active photonics. J. Semicond. 2018, 39, 061001. [CrossRef]

15. Zheng, J.; Liu, Z.; Xue, C.; Li, C.; Zuo, Y.; Cheng, B.; Wang, Q. Recent progress in GeSn growth and GeSn-based photonic devices. J. Semicond. 2018, 39, 061006. [CrossRef]

16. Lin, H.; Chen, R.; Lu, W.; Huo, Y.; Kamins, T.I.; Harris, J.S. Structural and optical characterization of SixGe1-x-ySny alloys grown by molecular beam epitaxy. Appl. Phys. Lett. 2012, 99, 141908. [CrossRef]

17. Kasper, E.; Werner, J.; Oehme, M.; Escoubas, S.; Burle, N.; Schulze, J. Growth of silicon based germanium tin alloys. Thin Solid Films 2012, 520, 3195. [CrossRef]

18. Kim, S.; Bhargava, N.; Gupta, J.; Coppinger, M.; Kolodzey, J. Infrared photoresponse of GeSn/n-Ge heterojunctions grown by molecular beam epitaxy. Opt. Express 2014, 22, 11029-11034. [CrossRef]

19. Taraci, J.; Zollner, S.; McCartney, M.R.; Menendez, J.; Santana-Aranda, M.A.; Smith, D.J.; Haaland, A.; Tutukin, A.V.; Gundersen, G.; Wolf, G.; et al. Synthesis of silicon-based infrared semiconductors in the Ge-Sn system using molecular chemistry methods. J. Am. Chem. Soc. 2001, 123, 10980. [CrossRef]

20. Margetis, J.; Mosleh, A.; Al-Kabi, S.; Ghetmiri, S.A.; Du, W.; Dou, W.; Benamara, M.; Li, B.; Mortazavi, M.; Naseem, H.A.; et al. Study of low-defect and strain-relaxed GeSn growth via reduced pressure CVD in H2 and N2 carrier gas. J. Cryst. Growth 2017, 463, 128. [CrossRef]

21. Wirths, S.; Buca, D.; Tiedemann, A.T.; Hollander, B.; Bernardy, P.; Stoica, T.; Grutzmacher, D.; Mantl, S. Reduced Pressure CVD Epitaxial Growth of Ge1-xSnx Using SnCl4 and Ge2H6. Meeting Abstracts (Electrochemical Society). In Proceedings of the ECS PRiME, Honolulu, HI, USA, 11 October 2012. Abstract Number 3214.

22. Senaratne, C.L.; Gallagher, J.D.; Jiang, L.; Aoki, T.; Smith, D.J.; Menendez, J.; Kouvetakis, J. Ge1-ySny (y = 0.01-0.10) alloys on Ge-buffered Si: Synthesis, microstructure, and optical properties. J. Appl. Phys. 2014, 116, 133509. [CrossRef]

23. Beeler, R.T.; Grzybowski, G.J.; Roucka, R.; Jiang, L.; Mathews, J.; Smith, D.J.; Menendez, J.; Chizmeshya, A.V.G.; Kouvetakis, J. Synthesis and Materials Properties of Sn/P-Doped Ge on Si(100): Photoluminescence and Prototype Devices. Chem. Mater. 2011, 23, 4480-4486. [CrossRef]

24. Margetis, J.; Yu, S.-Q.; Bhargava, N.; Li, B.; Du, W.; Tolle, J. Strain engineering in epitaxial Ge1-xSnx: A path towards low-defect and high Sn-content layers. Semicond. Sci. Technol. 2017, 32. [CrossRef]

25. Grant, P.C. GeSn Thin Film Epitaxy and Quantum Wells for Optoelectronic Devices. Ph.D. Dissertation, University of Arkansas, Fayetteville, AR, USA, December 2018.

26. Assali, S.; Attiaoui, A.; Atalla, M.R.M.; Dijkstra, A.; Kumar, A.; Mukherjee, S.; Abdi, S.; Moutanabbir, O. Epitaxial GeSn and its integration in MIR optoelectronics. In Proceedings of the 2020 Conference on Lasers and Electro-Optics (CLEO), San Jose, CA, USA, 10-15 May 2020.

27. Kim, Y.; Assali, S.; Burt, D.; Jung, Y.; Joo, H.-J.; Chen, M.; Ikonic, Z.; Moutanabbir, O.; Nam, D. Enhanced GeSn Microdisk Lasers Directly Released on Si. arXiv 2106, arXiv:08874.2021. [CrossRef]

28. Margetis, J. RPCVD Growth of Epitaxial Si-Ge-Sn Alloys for Optoelectronics Applications. Ph.D. Dissertation, Arizona State University, Phoenix, AR, USA, January 2018.

29. Wirths, S.; Buca, D.; Mussler, G.; Tiedemann, A.T.; Holländer, B.; Bernard, P.; Stoica, T.; Grützmacher, D.; Mantl, S. Reduced Pressure CVD Growth of Ge and Ge 1-x Sn x Alloys. ECS J. Solid State Sci. Technol. 2013, 2, 99-102. [CrossRef] 
30. Von Den Driesch, N.; Stange, D.; Wirths, S.; Mussler, G.; Holländer, B.; Ikonic, Z.; Hartmann, J.M.; Stoica, T.; Mantl, S.; Grützmacher, D.; et al. Direct Bandgap Group IV Epitaxy on Si for Laser Applications. Chem. Mater. 2015, 27, 4693-4702. [CrossRef]

31. Loo, R.; Shimura, Y.; Ike, S.; Vohra, A.; Stoica, T.; Stange, D.; Buca, D.; Kohen, D.; Margetis, J.; Tolle, J. Epitaxial GeSn: Impact of process conditions on material quality. Semicond. Sci. Technol. 2018, 33, 114010. [CrossRef]

32. Aubin, J.; Hartmann, J.M.; Gassenq, A.; Milord, L.; Pauc, N.; Reboud, V.; Calvo, V. Impact of thickness on the structural properties of high tin content GeSn layers. J. Cryst. Growth 2017, 473, 20-27. [CrossRef]

33. Aubin, J.; Hartmann, J.M.; Gassenq, A.; Rouviere, J.L.; Robin, E.; Delaye, V.; Cooper, D.; Mollard, N.; Reboud, V.; Calvo, V. Growth and structural properties of step-graded, high Sn content GeSn layers on Ge. Semicond. Sci. Technol. 2017, 32, 94006. [CrossRef]

34. Dou, W.; Alharthi, B.; Grant, P.C.; Grant, J.M.; Mosleh, A.; Tran, H.; Du, W.; Mortazavi, M.; Li, B.; Naseem, H.; et al. Crystalline GeSn growth by plasma enhanced chemical vapor deposition. Opt. Mater. Express 2018, 8, 3220-3229. [CrossRef]

35. Tran, H.; Du, W.; Ghetmiri, S.A.; Mosleh, A.; Sun, G.; Soref, R.A.; Margetis, J.; Tolle, G.; Li, B.; Yu, S.Q.; et al. Systematic study of Ge1-xSnx absorption coefficient and refractive index for the device applications of Si-based optoelectronics. J. Appl. Phys. 2016, 119, 103106. [CrossRef]

36. Grant, P.C.; Dou, W.; Alharthi, B.; Grant, J.M.; Tran, H.; Abernathy, G.; Mosleh, A.; Du, W.; Li, B.; Mortazavi, M.; et al. UHV-CVD Growth of High Quality GeSn Using SnCl4: From Growth Optimization to Prototype Devices. Opt. Mater. Express. 2019, 9, 3277-3291. [CrossRef]

37. Tseng, H.H.; Wu, K.Y.; Li, H.; Mashanov, V.; Cheng, H.H. Mid-infrared electroluminescence from a Ge/Ge0.922Sn0.078/Ge double heterostructure p-i-n diode on a Si substrate. Appl. Phys. Lett. 2013, 102, 182106. [CrossRef]

38. Kim, H.-W.; Shin, K.W.; Lee, G.-D.; Yoon, E. High quality Ge epitaxial layers on Si by ultrahigh vacuum chemical vapor deposition. Thin Solid Films 2009, 517, 3990-3994. [CrossRef]

39. Ye, H.; Yu, J. Germanium epitaxy on silicon. Sci. Technol. Adv. Mater. 2014, 15, 024601. [CrossRef] 